\title{
Sonographic Features of Salivary Gland Tumours
}

\author{
Mohi Al-Tamami ${ }^{a}$ Nail Al-Naqeeb ${ }^{b}$ Agnes Kovacs $^{a}$ \\ Hussein Dashtic John Madda Patrick ${ }^{\mathrm{d}}$ \\ Departments of a Clinical Radiology and bSurgery, Al-Amiri Hospital, cDepartment of \\ Surgery, Faculty of Medicine, Kuwait University, and dUnit of Histopathology, Kuwait
}

\section{Key Words}

Salivary tumours · Ultrasonography

\begin{abstract}
Objective: High resolution ultrasonography (US) has played a significant role in the study of salivary gland (SG) pathology and has surpassed sialography in the study of SG tumours. This report discusses the sonographic features of SG tumours examined during the last 5 years. The value of these features as diagnostic indicators of the nature (benign or malignant) and histotype of these tumours is assessed. Methods: High resolution ultrasonography was used in the study of 83 cases of salivary tumours, 78 of which were of the parotid gland and 5 of the submandibular gland. Sixty-six $(80 \%)$ of these tumours were benign. An US scanner with a 7.5-MHz real-time linear probe was used. The diseased SG was examined in mul-
\end{abstract}

\begin{tabular}{ll}
\hline KARGER & (1) 1999 S. Karger AG, Basel \\
1011-7571/99/0083-0201\$17.50/0 \\
Fax +4161306 1234 & \\
$\begin{array}{l}\text { E-Mail karger@karger.ch } \\
\text { www.karger.com }\end{array}$ & $\begin{array}{l}\text { Accessible online at: } \\
\text { http://BioMedNet.com/karger }\end{array}$
\end{tabular}

tiple planes to fully delineate and locate the lesions and to characterize their sonographic features. Results: US detected and correctly located all tumours. The sonographic features of the various tumour categories studied are presented with special emphasis on diagnostically significant ones. The value of these features as diagnostic indicators of the nature (benign or malignant) and the histotype of these tumours is assessed. In this study, the rate of correct US identification of tumour benignity was $100 \%$ in a group of 15 adenolymphomas and 12 benign non-epithelial tumours, and $94 \%$ in a group of 34 pleomorphic adenomas. Five cases of recurrent pleomorphic adenoma were also studied. Correct US identification of malignancy was achieved in $82 \%$ of malignant tumours (15 carcinomas and 2 isolated primary nonHodgkin's lymphomas of the parotid gland). US identification of the specific tumour histotype was quite successful in the case of be-
Dr. Mohi Al-Tamami, MD, PhD

Clinical Radiology Department

Al-Amiri Teaching Hospital

PO Box 4077, 13041 Safat (Kuwait)

Tel. +965 2467498, Fax +9652447584 
nign tumours with an accuracy of $84 \%$ in pleomorphic adenoma, 93\% in adenolymphoma, and $100 \%$ in vascular tumours and lipoma. Only 1 of the 17 malignant tumours (a carcinomatous pleomorphic adenoma) was specifically identified. Conclusion: Based on the diagnostic capabilities of US revealed in this study as well as its operational advantages, US is strongly recommended as the first-line imaging procedure for all masses at the SG regions, to be followed by US-guided fine needle aspiration biopsy particularly for equivocal cases.

\section{Introduction}

High resolution ultrasonography (US) has played a significant role in the study of salivary gland (SG) pathology and has surpassed sialography in the study of SG tumours [113]. During the last 5 years high resolution real-time US was used as the first-line investigation procedure for all forms of SG disorders at the Al-Amiri Hospital, Kuwait. This report discusses the sonographic features of SG tumours examined during this period. The value of these features as diagnostic indicators of the nature (benign or malignant), and the histotype of these tumours are assessed.

\section{Subjects and Methods}

A group of 83 patients (42 males and 41 females) whose ages ranged from 6 months to 80 years (mean 35 years) was examined. These patients were referred randomly from the outpatient surgical clinic with some of the SG disorders. An US scanner (Toshiba Sonolayer) with a $7.5-\mathrm{MHz}$ real-time linear probe was used with a standoff pad in every case. The diseased SG was examined in multiple planes to fully delineate and locate the lesions and to characterize their sonographic features. US examination was extended to the rest of the SG regions as well as to the entire neck. US features ana- lysed for each tumour included: tumour size, location, echodensity, tumour margins, internal echotextural pattern, contour features, tumour calcification, sound transmission and evidence of extraglandular tumour spread. Following US examination, US-guided fine needle aspiration biopsy (FNAB) was performed for all positive cases. A histopathological diagnosis was obtained for cases which were operated on. Operations were performed on 68 patients according to locally established techniques $[14,15]$.

\section{Results}

Table 1 presents the various salivary tumour categories and subcategories examined by high resolution US.

\section{Sonographic Features of Benign Tumours}

\section{Pleomorphic Adenoma (Mixed Parotid \\ Tumour)}

Of the 39 cases of pleomorphic adenoma (PA) that were studied, 36 were in the parotid gland (PG) and 3 in the submandibular gland (SMG). Remarkably 30 cases were on the right side. One of the patients had a PA in one of the PGs and an acinic cell tumour in the other PG. Thirty-three (92\%) of the 36 PAs in the PG originated from its superficial lobe and the rest from its deep lobe. Maximum US diameter ranged from 1.3 to $6.0 \mathrm{~cm}$ (mean $2.7 \mathrm{~cm}$ ).

All PAs appeared as hypoechogenic solid tumour masses with an internal echopattern varying from homogeneous to slightly nonhomogeneous (fig. 1a-f). Small cystic degenerative areas were occasionally seen (fig. 1e). Coarse tumour calcification was seen in one PA of the SMG (fig. 1f). Contour lobulation was a prominent feature in the majority of PAs and was either general contour lobulation or segmental with polypoid structure formations (fig. 1a-e). 
Table 1. Histologic identity of SG tumours studied by real-time high resolution US

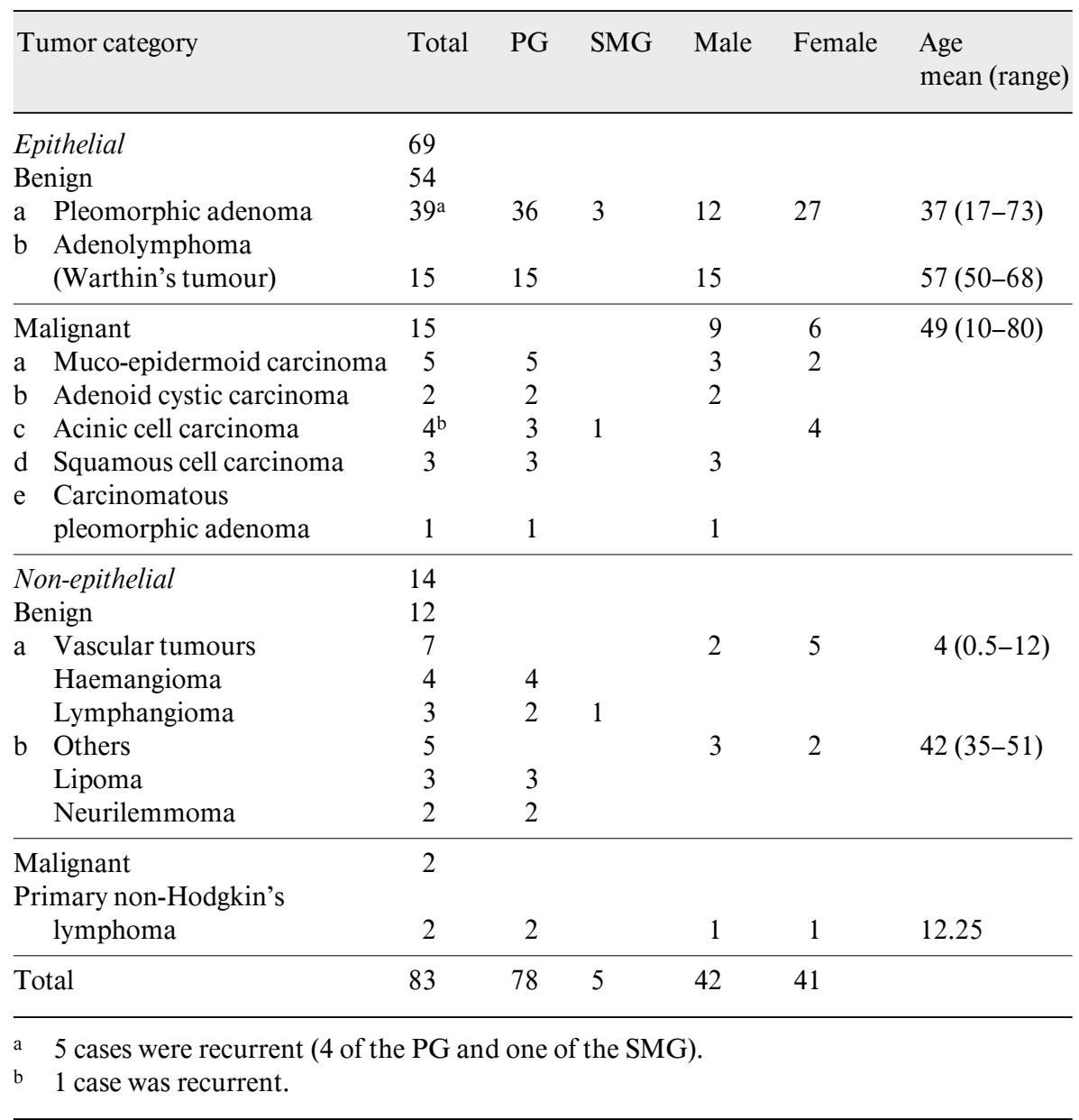

Five of the PAs studied were recurrent (4 $P G$ and 1 SMG) and their presentation differed from the pre-operative ones. All preoperative tumours presented as solitary tumours and $94 \%$ of these tumours had a sharp margin. Only 2 cases showed ill-defined margins, which after histology was found to be due to inflammatory changes around the PA.

The recurrent PAs presented as multiple nodular tumour implants inside and/or outside the SGs. Many of the recurrent PA im- plants showed irregular margins with sharp extensions reaching and involving the skin in 1 case (fig. $1 \mathrm{~g}-\mathrm{i}$ ). A prominent feature of all PAs was increased sound transmission with strong acoustic enhancement distal to the tumour. Sonographically homogeneous PAs had more epithelial and less mesenchymal elements than heterogeneous ones. Chronic parotid inflammation was occasionally seen in the involved PG. 

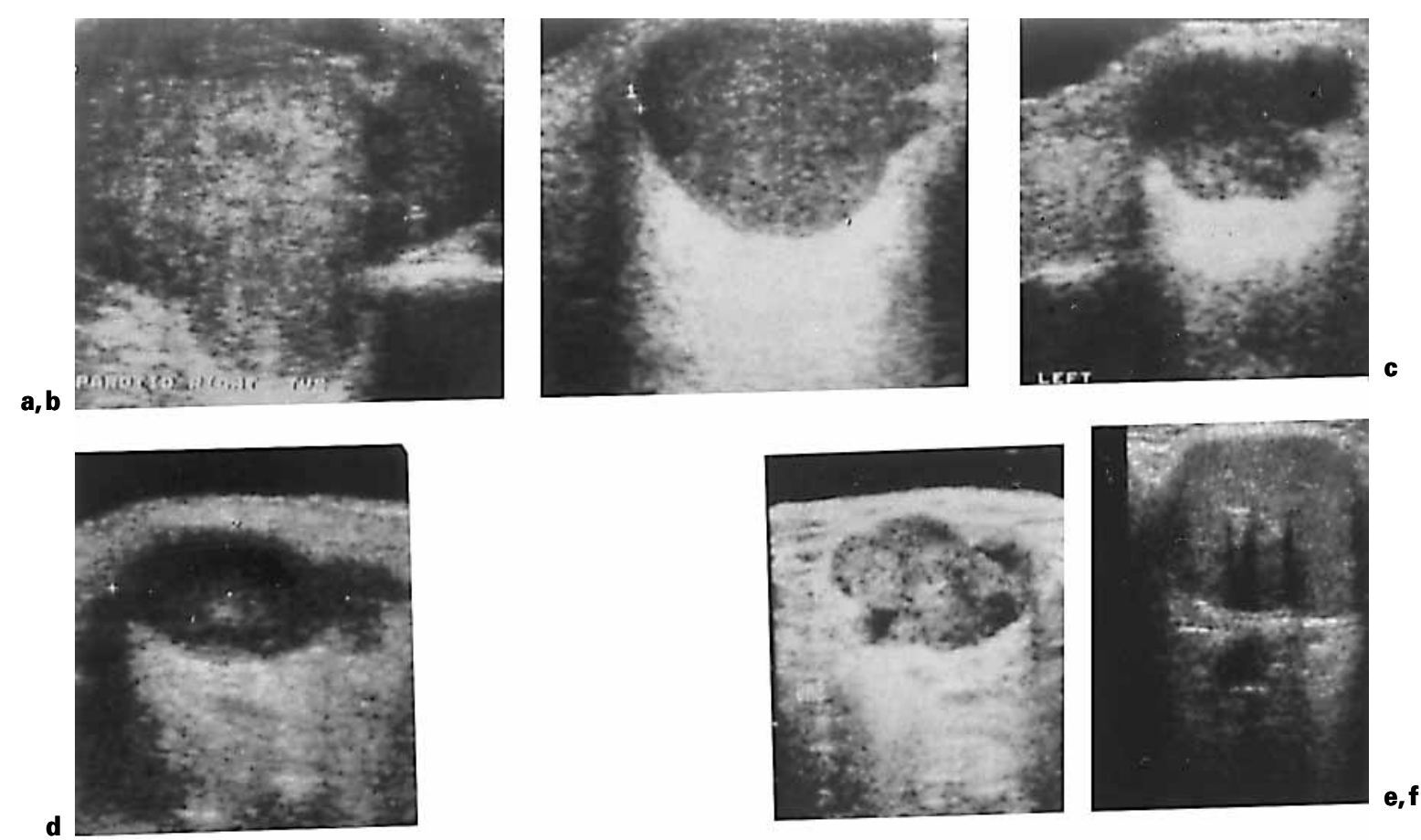

$\mathbf{g}, \mathbf{h}$
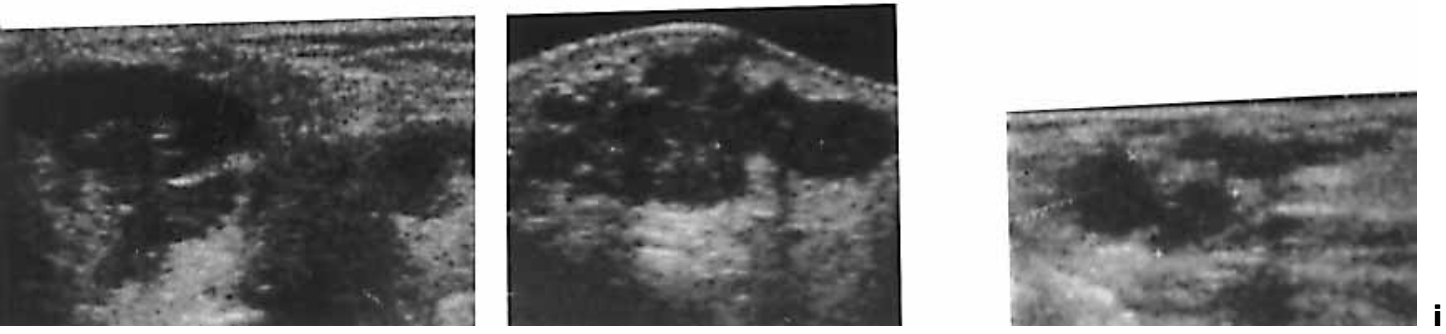

Fig. 1. a-d PG. PA-characteristic prominent general and segmental contour lobulations as well as strong distal acoustic enhancement. e, f SMG: prominent contour lobulations and small degenerative cystic area (e) and coarse focal central calcifications (f). $\mathbf{g - i}$ Recurrent PA: PG $(\mathbf{g}, \mathbf{h})$ and SMG (i). Intra- and extraglandular irregular nodular tumour implants.

Adenolymphoma (Warthin's Tumour)

Characteristically all 15 cases of Warthin's tumour (WT) were in the PG, and in male patients above 50 (mean 57) years of age. A common clinical feature of WT was the recurrent episodes of inflammation. Four of our patients with WT were clinically thought to have parotid abscesses. Ten cases of WT were unilateral and 5 were bilateral with 3 of these being multicentric as well. Bilateral multicentric tumour nodules measured between 0.8 and $2.5 \mathrm{~cm}$ in maximum diameters. Unicentric tumours tended to be larger with maximum diameters ranging from 3.8 to $5.6 \mathrm{~cm}$. 

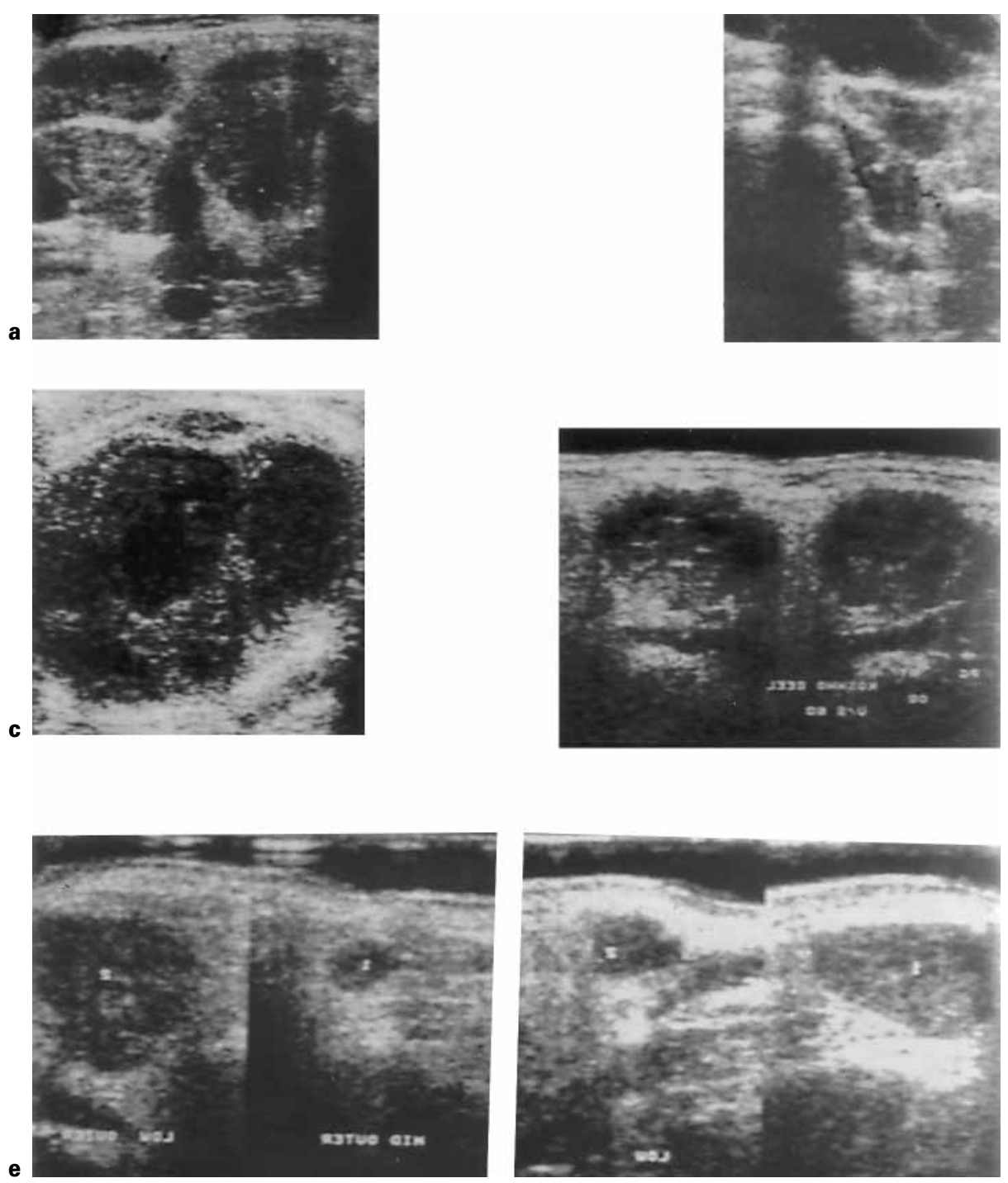

Fig. 2. a, b Unilateral unicentric: large complex cystic mass with large multiloculations. c Infected unilateral unicentric WT: large multiloculated with distorted echotexture and necrotic areas. d Bilateral unicentric: one complex mass in each PG with barely differentiated cystic components. e Bilateral multicentric: two pseudosolid masses in each PG consisting of very tiny cystic components.

All WTs appeared as hypoechogenic tumour masses with well-defined contours. The internal tumour echopattern varied with the size of the tumour nodule. Large unicentric tumours presented as large multiloculated thickly septated cystic mass (fig. 2a, b). One of the large tumours protruded beyond the surface contours of the PG. Large WTs, especially those extending beyond the confines of the PG, were managed surgically. Gross appear- 

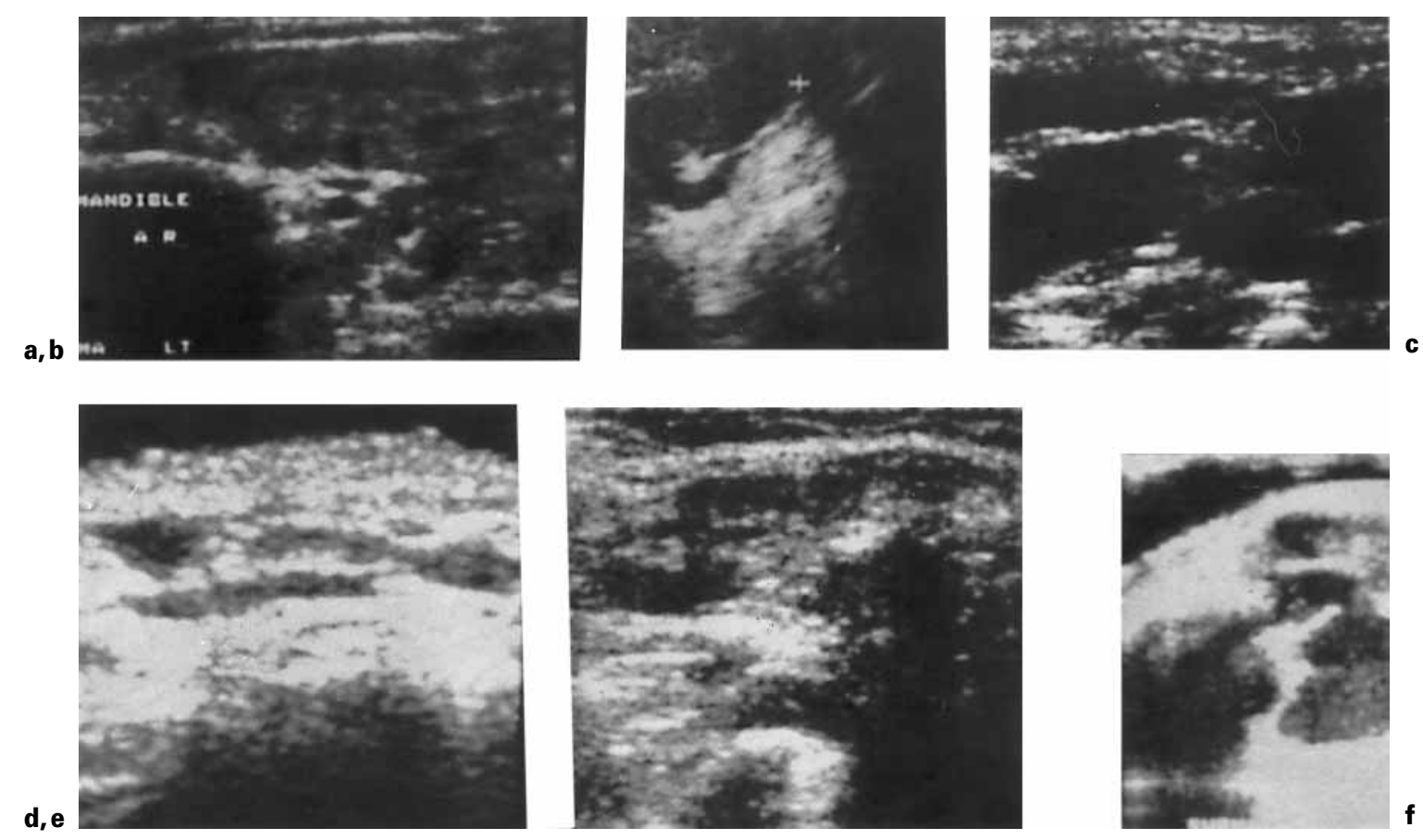

Fig. 3. Vascular tumours. a-c Haemangioma (PG): honeycomb-looking diffuse haemangiomatous involvement of the whole gland (a); intraparotid partly septated unilocular cystic haemangioma (b); large multilocular cystic structure (c). d-f Lymphangioma: superficial multitubular worm-like spaces (d), superficial small multilocular cystic spaces, both involving the superficial layers of the PG (e), and large shapeless multiloculated cystic structure replacing the SMG (f).

ance of the surgical specimen matched with the echofeatures of the tumour.

Severely inflamed WTs retained the sharp margins and had distorted echopattern with necrotic foci (fig. 2c). Small multicentric tumour nodules gave a pseudosolid internal echopattern which was found to be formed of thinly septated tiny cystic structures on using a higher frequency US (fig. $2 \mathrm{~d}$, e). The gross pathological structure of WTs matched their echotexture.

\section{Vascular Tumours (Haemangiomas and Lymphangiomas)}

One case of capillary and 3 cases of cavernous haemangiomas of the PG were seen. The capillary haemangioma had a characteristic honeycomb echopattern and diffusely involved the gland (fig. 3a). One of the cavernous haemangiomas appeared as an intraparotid cyst having an incomplete septum and very fine low level echoes (fig. 3b). The other two haemangiomas were large and extended beyond the confines of the gland. In 1 patient the cheek and upper lip were also involved whereas in another the whole ipsilateral side of the head, face and neck were also involved. 
Fig. 4. a, b Lipoma of the PG presenting as encapsulated oval hypoechogenic solid masses traversed by thin linear echogenic strands.
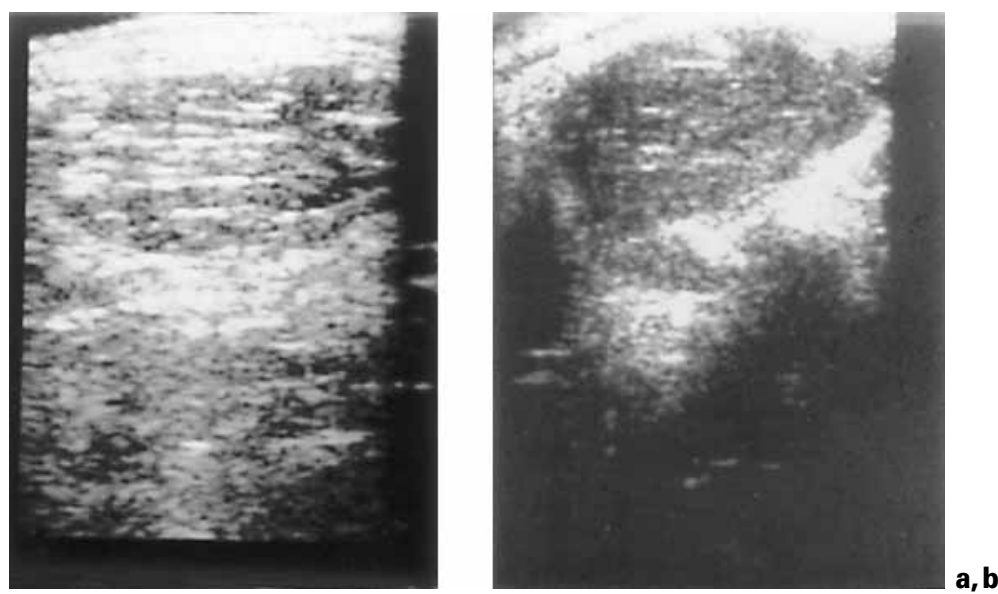

In both cases the masses were large, easily compressible, shapeless, multiloculated cystic structures. Some of the locules had phleboli (fig. 3c).

Two cases of parotid lymphangioma were seen and presented with compressible fullness over the gland. Both cases involved the outer layers of the superficial lobe with minimal extraparotid extension. Sonographically, one showed as multiple tubular worm-like structures (fig. 3d) and the other as multiple small cystic locules (fig. 3e). No signal was obtained on Doppler examination of both cases.

One upper cervical cystic lymphangioma involved one of the SMGs turning it into a large, shapeless, compressible mass with thinwalled locules of varying sizes and shapes (fig. 3f).

\section{$P G$ Lipomas}

All 3 cases investigated had similar sonographic pictures. They were compressible, generally hypoechogenic solid masses traversed by thin linear echoreflective strands. They were easily differentiated from the parotid parenchyma (fig. $4 a, b$ ).

Sonographic Features of Salivary Gland Tumours

\section{Neurogenic Tumours}

Two cases intraparotid neurilemmomas were studied and appeared as well-defined, uniformly hypoechogenic masses with diffusely scattered very tiny echoreflective foci (fig. 5a, b).

\section{Sonographic Features of Malignant SG Tumours}

There were 15 carcinomas and 2 primary non-Hodgkin's lymphomas. Fourteen cases of carcinoma were seen in the PG and 1 in the SMG. One of the carcinomas developed from a non-operated pleomorphic adenoma of 20 years' duration in an 80-year-old man. All malignant tumours were hypoechogenic when compared to normal salivary gland parenchyma. Malignant lesions show acoustic enhancement under necrotic areas (fig. 6a) and if partial malignant transformation occurred (fig. 6c) the benign lesions did not show acoustic shadowing.

Three categories of US features of carcinomas varying with the stage of malignancy at the time of presentation were seen: the first

Med Principles Pract 1999;8:201-212 
Fig. 5. a, b PG neurolemmoma: well-defined hypoechogenic solid masses with diffusely scattered very tiny echogenic foci.
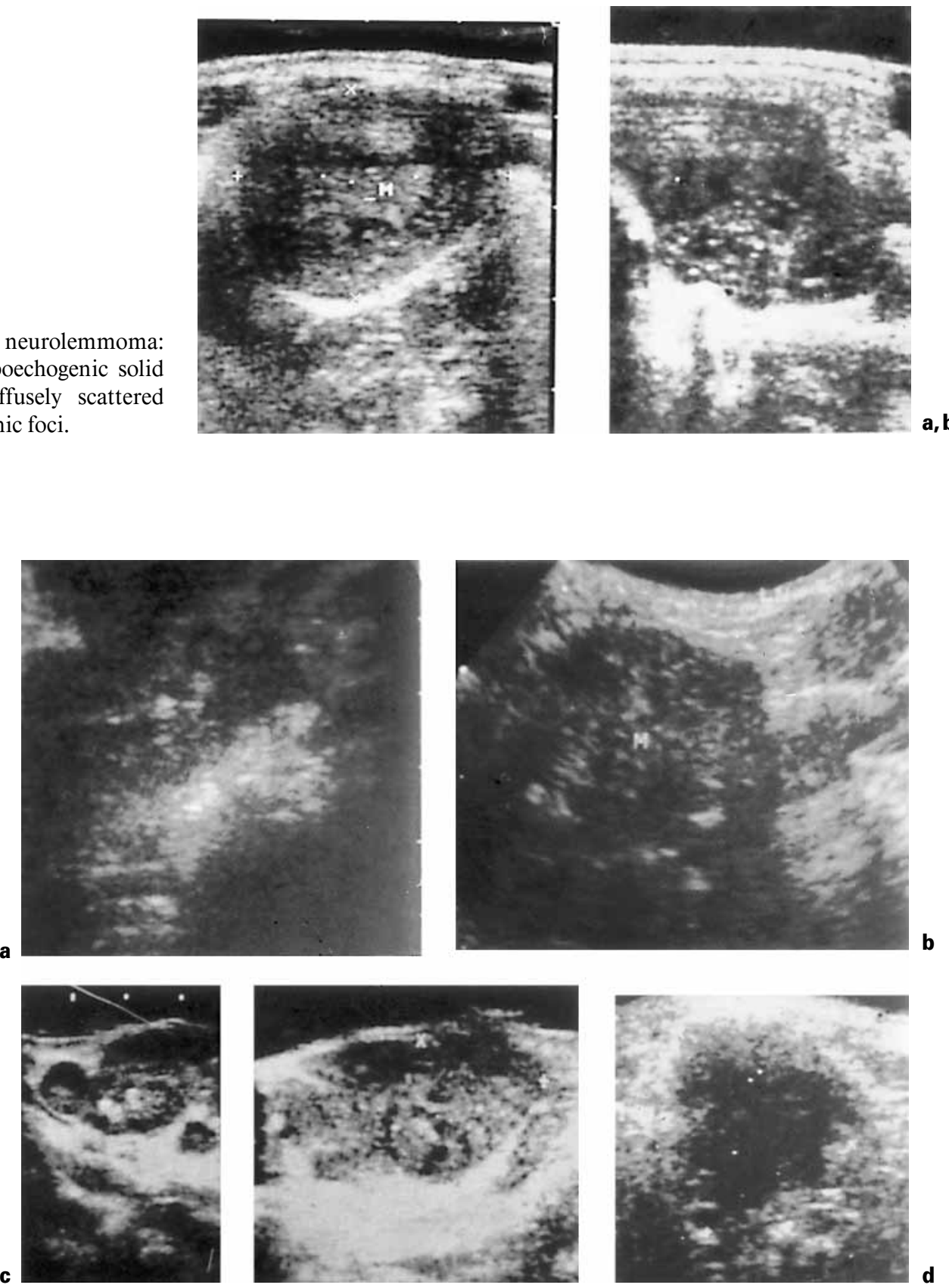

Fig. 6. Carcinoma (CA). a-d Advanced CA. Irregular/hazy margins, heterogeneous internal echopattern, distal acoustic shadowing, extraglandular spread: PG squamous cell CA with necrosis and direct extension (a); SMG acinic cell CA with microcalcifications and lymph node metastasis (b); PG carcinomatous PA with necrosis, skin invasion, intraparotid lymph node involvement and partly PA-characteristic sharp contour lobulations and coarse-type calcifications (c); PG adenoid cystic CA with skin invasion (d). e, f Locally invasive CA. 
category included advanced carcinomas (fig. 6a-d) and was characterized by hazy invasive tumour margins, heterogeneous internal echopattern with necrosis and/or tumour calcification, varying degrees of distal acoustic shadowing and US evidence of direct and/ or lymphatic tumour spread. The second category included early invasive carcinomas (fig. 6e, f) and was characterized by hazy invasive tumour margins with otherwise homoge- neous internal echopattern, distal acoustic enhancement and no evidence of extraglandular tumour spread. The third category included low grade carcinomas at a pre-invasive stage (fig. 6g, h) and was characterized by sharp tumour margins, homogenous internal echopattern and uniform distal acoustic enhancement.

Two cases of isolated primary parenchymal non-Hodgkin's lymphoma of the PG
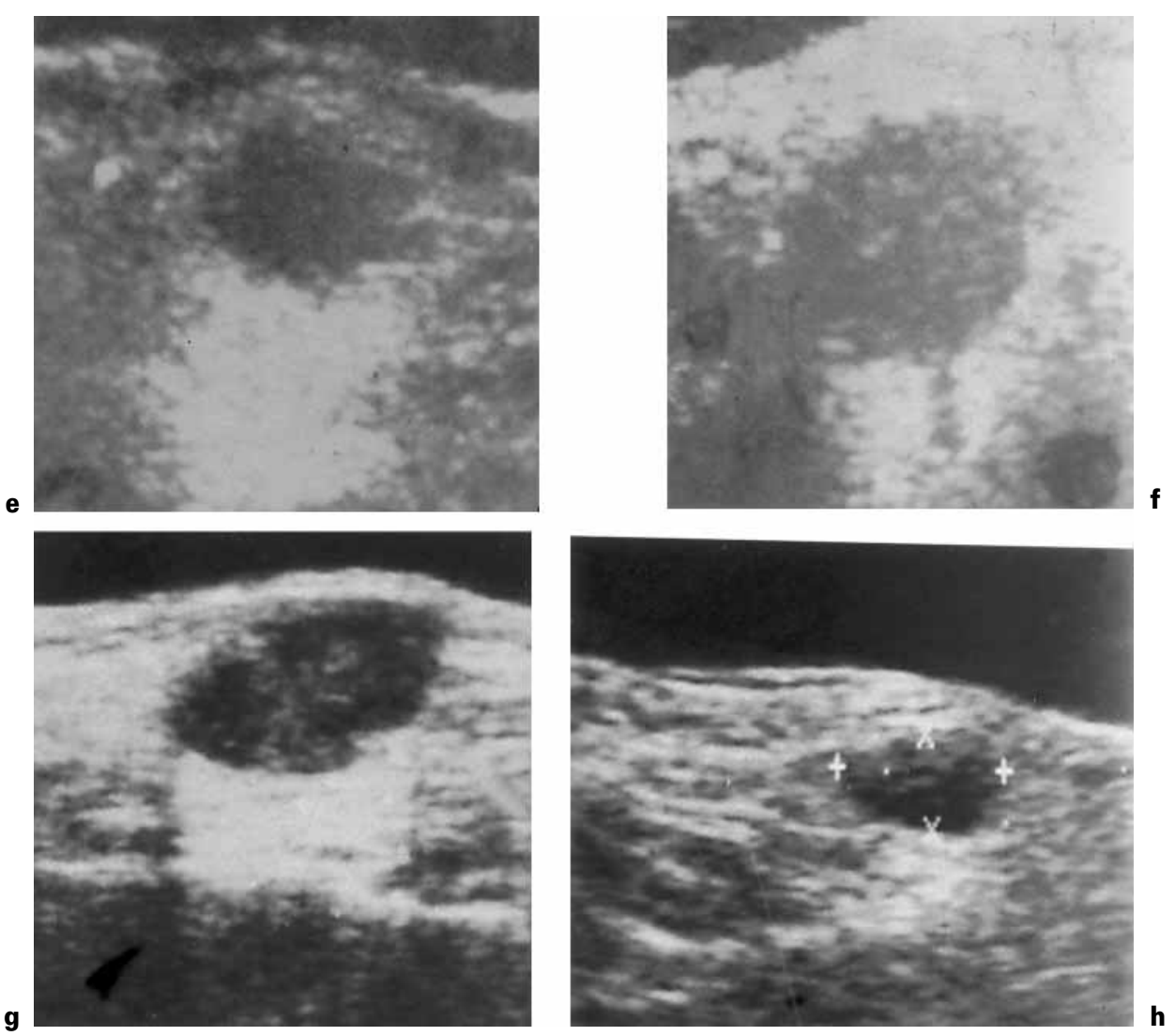

Irregular margins, homogeneous internal echopattern, distal acoustic enhancement: PG recurrent acinic cell CA (e) and muco-epidermoid CA with long finger-like extensions into normal parenchyma (f). g, h Low grade pre-invasive CA. Benign-like features with sharp margins, homogeneous internal echopattern, distal acoustic enhancement: PG acinic cell CA (g) and PG muco-epidermoid CA (h). 

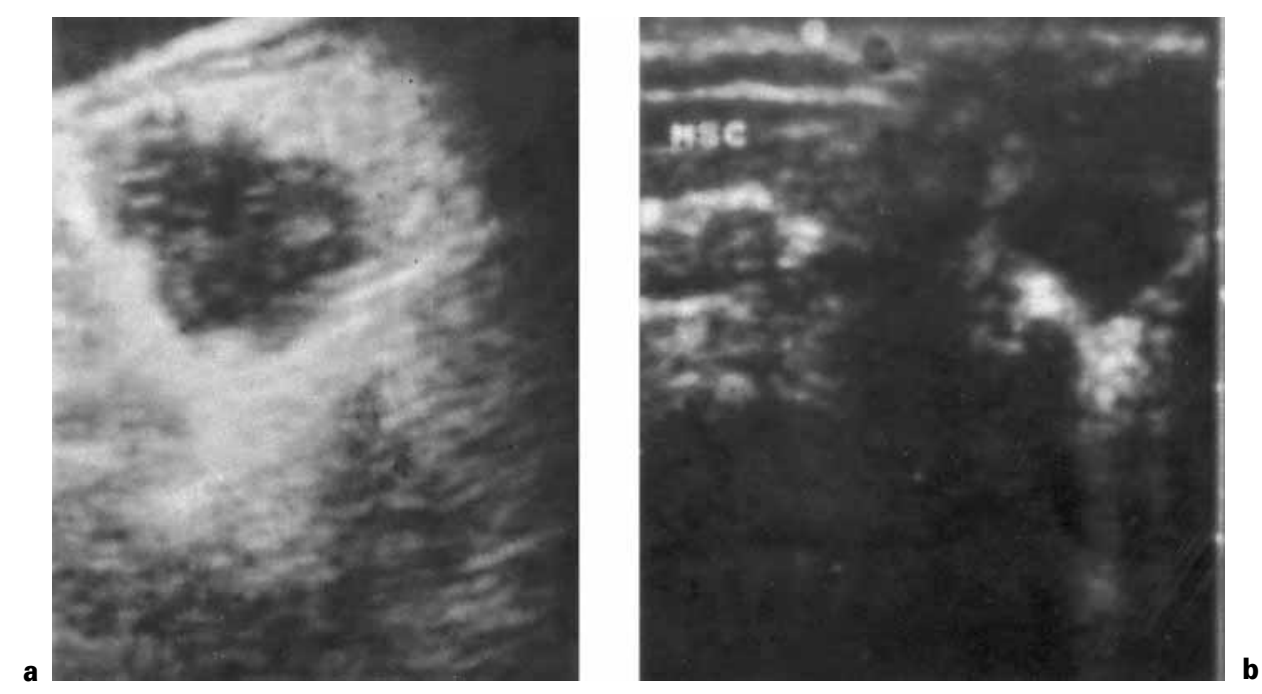

Fig. 7. PG primary non-Hodgkin's paranchymal lymphoma. a Locally invasive. b With extraparotid extension.

(fig. 7a, b) were not seen to be associated with involvement of the parotid lymph nodes and other lymphomatous lesions in the body. One case (fig. 7a) presented as solitary intraparotid nodular lesion having shaggy invasive margins with otherwise homogeneous echopattern and distal acoustic enhancement. The other (fig. 7b) was multinodular and more invasive with direct extension into adjacent paraparotid connective tissues and muscles.

\section{Discussion}

The vast majority of SG tumours arise from the PG and are usually treated surgically $[14,15]$. Since PG surgery presents a considerable challenge, particularly in facial nerve injury, all questions related to the differential diagnosis and the plan of management of the PG swellings should, as far as possible, be fully assessed pre-operatively.
US detected and correctly located all tumours in the current study including those that were small non-palpable and deeply located, and also multicentric and bilateral PG tumours. Acutely inflamed adenolymphoma clinically misdiagnosed as parotid abscess as well as non-neoplastic PG swellings (hyperplastic PG lymph nodes, parotid cysts and granulomatous masses) were correctly identified sonographically. Similarly, parasalivary gland masses, most often swollen lymph nodes and occasionally congenital cysts or mesenchymal tumours, were correctly located and identified. The mentioned lesions were seen in patients other than those reported except for 1 case of PA combined with a parotid cyst in the same gland. The mentioned lesions were clinically thought to be SG tumours and will be reported later separately.

All SG tumours, whether benign or malignant, were hypoechogenic compared to normal SG parenchyma. In most cases it was pos-

Al-Tamami/Al-Naqeeb/Kovacs/Dashti/ Patrick 
sible to determine whether the SG tumour was benign or malignant using US. The characteristic features of benign tumours were sharp tumour margins, associated with fairly uniform solid internal echopattern in cases of solid tumours like PAs, lipomas and neurogenic tumours. Tumours with complex internal structure such as adenolymphoma and vascular tumours had well-organised complex echopattern. All benign tumours also showed increased sound transmission with distal acoustic enhancement of degrees varying according to the US transmission properties of the tumour tissues and its structural design.

The benign nature of all $(100 \%)$ non-epithelial tumours and adenolymphomas was accurately predicted by US. It was slightly less accurate (94\%) accuracy) in 'primary' (nonrecurrent) PAs. Two cases out of the 34 PAs were inaccurately assigned to the malignant group because of hazy borders caused by adjacent inflammatory reaction. Gritzman [6] reported a $94 \%$ US identification rate for benignity of salivary tumours above $1 \mathrm{~cm}$ in size. He pointed out that borders of tumours less than $1 \mathrm{~cm}$ were not clear enough to detect their nature. Yasumoto et al. [16] reported an accuracy of $95.7 \%$ in assessing the benignity of SMG tumours. In their study 1 of the 21 PAs found in the SMG was recurrent and showed irregular margins. In the present study all 5 recurrent PAs presented as irregular multinodular tumour implants within the SGs and/or as extrinsic mass.

Most (82\%) of the malignant tumours (carcinomas and non-Hodgkin's lymphomas) had hazy invasive margins. Few (18\%) had sharp margins and these proved to be low grade preinvasive muco-epidermoid and acinic cell tumours. Gritzman [6] using unsharp tumour margins as an indicator of malignancy correctly identified malignancy in $72 \%$ of the cases, while $28 \%$ of the malignant tumours in his study had sharp margins.

Sonographic Features of Salivary Gland Tumours
A smaller incidence of malignant tumours with sharp margins was reported by Yasumoto et al. [16] in their study of SMG tumours. One of the $12(8.5 \%)$ malignant SMG tumours (an adenoid cystic carcinoma) showed sharp US margins and also had a smooth pseudocapsule on gross pathological examination.

Limitations in identifying the true nature of some tumours are also encountered with CT and MRI [16, 20]. To overcome such limitations FNAB [20, 21] is resorted to. An USguided FNAB is recommended for puncturing small non-palpable or deeply located lesions as well as particular regions in partly necrotic and complex cystic lesions. In our study three stages of malignancy could be sonographically characterized: an advanced stage constituting $47 \%$, an early invasive stage which constituted $33 \%$ and a low grade preinvasive stage seen in $20 \%$ of the cases.

Sonographic identification of extraglandular direct and/or local lymph node spread was possible in all malignant SG tumours presenting in an advanced stage. CT or MRI is however needed for full staging of advanced malignancy with suspected invasion of deep tissues or bone. CT or MRI might also be needed for complete delineation of large deep lobe tumours (the anterior part of which might be masked by the acoustic shadowing of the overlying mandibular bone) and differentiating these from parapharyngeal tumours. Since at least $90 \%$ of PG tumours arise from the superficial lobe and $80 \%$ are benign, the need for CT or MRI is usually limited. In this study CT was used for full staging of only advanced squamous cell carcinomas involving the deep lobe.

Identification of the specific histologic type of tumours in this study, using a combination of sonographic and clinical evaluation, was successful in the majority of benign tumours with an accuracy of $100 \%$ for vascular

Med Principles Pract 1999;8:201-212 211 
tumours and lipomas, $93 \%$ for adenolymphomas and $80 \%$ for PA. Specific identity of only one malignant tumour (carcinoma in a PA) was predicted. This identification was primarily based on the presence of characteristic US features of PA in the part of the tumour which did not undergo malignant transformation.

Other malignant tumours that showed some special features were too few to be of specific significance. For instance, 2 out of 5 muco-epidermoid carcinomas showed peculiar elongated finger-like tumour extension into the surrounding PG tissues. Tumour necrosis was particularly prevalent in squamous cell carcinomas and carcinomatous pleomorphic adenoma. Tumour microcalcification was seen only in an advanced SMG acinic cell carcinoma while coarse tumour calcification was seen in carcinomatous PA.

\section{References}

1 Ballereni G, Mantero M, Sbrocca M: Ultrasonic patterns of parotid masses. ICU 1984;12:273-277.

2 Barlett LJ, Pon M: High resolution real time ultrasonography of the submandibular glands. J Ultrasound Med 1984;3:433-437.

3 Bruneton JN, Sicart M, Roux P, Pastand P, Nicolan A, Delorme G: Indications for ultrasonography in parotid pathologies. Rofo Fortschr Geb Röntgenstr Neuen Bildgeb Verfahr 1983;138:22-24.

4 Bruneton JN: Ultrasonography of the Neck. Berlin, Springer, 1987, pp 64-80.

5 Derehi LE, Sobliati L: Salivary glands; in Cosgrove $\mathrm{D}$, Meire $\mathrm{H}$, Dewbery K (eds): Abdominal and General Ultrasound. London, Churchill Livingstone, 1993, vol 2, pp 677-693.

6 Gritzman N: Sonography of the salivary glands. AJR 1989;153:161166.

7 Luyk NH, Doyle T, Ferguson MM: Recent trends in imaging the salivary glands (review). Dentomaxillofac Radiol 1991;20:3-10.
8 Seibert RW, Seibert JJ: High resolution ultrasonography of the parotid gland in children. Pediatr Radiol 1986;16:374-379.

9 Solbiati L, Cioffl V, Ballarati E: Ultrasonography of the neck. Radiol Clin North Am 1992;30:941-954.

10 Wittich GR, Scheible WF, Hajek PC: Ultrasonography of the salivary glands. Radiol Clin North Am 1985; 23:29-37.

11 Akin I, Esmer N, Gereeker M, et al: Sialographic and ultrasonographic analysis of major salivary glands. Acta Otolaryngol (Stockh) 1991; 111:600-606.

12 Da-Xi S, Hai-Xiong S, Qiang Y: The diagnostic value of ultrasonography and sialography in salivary gland masses. Dentomaxillofac Radiol 1987;16:37-45.

13 Zbaren P, Ducommun JC: Diagnosis of salivary gland disease using ultrasound and sialography: A comparison. Clin Otolaryngol 1989;14: 189-197.

14 Al-Naqeeb N, Meyza J, Ali M: The management of salivary gland swellings in Kuwait. J R Coll Surg Edinb 1985;30:227-230.

15 Al-Naqeeb N, Dashti H, Al-Muhanna A, Behbehani A: Parotid gland tumours: A 15 year experience. J R Coll Surg Edinb 1992;37:89-93.
16 Yasumoto M, Shibya H, Suzuki S, Ishi J, Amagara T, Ida M, Okada N: Computed tomography and ultrasonography in submandibular tumours. Clin Radiol 1992;46:114120.

17 Rabinov K, Kell T, Gordon Ph: CT of the salivary glands. Radiol Clin North Am 1984;22:145-159.

18 Ellen KT, Hugh IC: MR of the salivary glands. Radiol Clin North Am 1989;27:379-391.

19 White AM, Byrne JW: A comparison of comuted tomography and ultrasound in the assessment of parotid masses. Clin Radiol 1987;38: 339-343.

20 Grazioli L, Olivetti L, Matricardi L, Zanetti U, Burlini D, Negrini S, Fugazzola $\mathrm{C}$, Chiesa $\mathrm{A}$ : Comparison of ultrasonography, CT and MRI in the assessment of parotid masses. Eur Radiol 1994;4:268-280.

21 Owen ERTC, Baner Jee AK, Richard AJN, et al: Role of fine needle aspiration cytology and computed tomography in the diagnosis of parotid swellings. Br J Surg 1989;76: 1273-1274. 